\title{
ATENCIÓN A LA DIVERSIDAD Y ESCUELA INCLUSIVA: LAS ACTITUDES DEL FUTURO PROFESORADO
}

\author{
María J. Hernández-Amorós \\ josefa.hernandez@ua.es \\ María E. Urrea-Solano \\ Aitana Fernández-Sogorb \\ María del Pilar Aparicio-Flores \\ Universidad de Alicante
}

Fecha de Recepción: 25 Marzo 2018

Fecha de Admisión: 10 Abril 2018

\section{RESUMEN}

El diseño y desarrollo de prácticas inclusivas se considera una de las líneas de actuación básicas para la construcción de una escuela para todos/as. Ambas acciones dependen en buena medida de las actitudes del profesorado, pudiendo estas verse modificadas por la formación que reciben. En este marco, esta investigación se planteó con el objetivo de conocer posibles diferencias en las actitudes de los futuros docentes de Educación Infantil y Primaria, y Educación Secundaria, así como de analizar la existencia de tales diferencias en el impacto que la formación puede tener sobre ellas. Se contó con la participación de 46 y 50 estudiantes, matriculados en el último curso de los Grados de Maestro en Ed. Infantil y Ed. Primaria y en el Máster de Profesorado de Ed. Secundaria, respectivamente, de la Facultad de Educación, de la Universidad de Alicante. Se realizó un estudio descriptivo y no paramétrico (prueba $U$ de Mann-Whitney), con el soporte del programa de análisis de datos SPSS .21. De los resultados se deduce que, en general, el profesorado en formación posee actitudes positivas hacia la diversidad y el principio de inclusión, aunque no están tan definidas en los ítems más controvertidos. Asimismo, destaca el impacto favorable que consideran tiene la formación sobre la sensibilización e interés por este tema. Sin embargo, se aprecia que los estudiantes de Grado poseen actitudes ligeramente más positivas que los de Máster, existiendo diferencias estadísticamente significativas en la consideración de la diversidad como elemento de riqueza y en el impacto de sus estudios sobre el interés por la formación al respecto. Se concluye que resulta apropiado continuar incidiendo sobre el plano actitudinal, en relación a esta temática, en tales titulaciones - con especial énfasis en el Máster -, así como valorar cualitativamente las diferencias que se han hallado entre los grupos estudiados.

Palabras clave: atención diversidad; inclusión; profesorado en formación; actitudes 


\title{
ATENCIÓN A LA DIVERSIDAD Y ESCUELA INCLUSIVA: LAS ACTITUDES DEL FUTURO PROFESORADO
}

\begin{abstract}
Attention to diversity and the inclusive school: the attitudes of pre-service teachers.

The design and development of inclusive practices is seen as one of the fundamental action areas in building a school for everyone. Both actions depend to a large extent on teachers' attitudes, since these can be modified by the training they receive. The aim of this study is to find out whether there are any differences in attitudes between future infant and primary and future secondary school teachers, and then analyse whether the existence of such differences affects the impact that training may have on them. The sample comprised 46 and 50 students enrolled in the final year of the Degree in Preschool and Primary Education and the Master's in Secondary Education respectively, in the Faculty of Education at the University of Alicante. A descriptive and non-parametric (Mann-Whitney $\mathrm{U}$ test) study was carried out with the help of SPSS .21 data analysis software. It can be deduced from the results that, generally speaking, teachers in training have positive attitudes towards diversity and the principle of inclusion, although attitudes are not so clear-cut in the more controversial areas. They also believe that training has a favourable impact on increasing awareness and interest in the subject. However, the degree students have slightly more positive attitudes than the master's students, and there are statistically significant differences when it comes to considering diversity as an element of richness and the impact that the course has on interest in training on the subject. Our conclusion is that it would be best to continue to work to change attitudes in this area and on these courses - especially the master's - and to qualitatively evaluate the differences between the groups studied.
\end{abstract}

Keywords: attention to diversity; inclusion; teachers in training; attitudes

\section{ANTECEDENTES}

La configuración de nuevas estructuras sociales - que deviene del proceso de globalización -, y su impacto a escala cultural, ha puesto en tela de juicio los sistemas tradicionales de enseñanza. Por ello, en los últimos años, se ha constatado que la escuela tiene ante sí el reto de educar para un mundo radicalmente distinto al que habitábamos. Los gobiernos internacionales, con este propósito, han tratado de impulsar políticas educativas centradas en favorecer un cambio metodológico, cuyo objetivo sea el desarrollo de personas autónomas y competentes que aprenden de forma permanente en una sociedad plural que cambia de manera incesante (Valle y Manso, 2013).

Esta apuesta por la transformación metodológica empieza a fraguarse en los procesos de formación inicial del profesorado, que son el punto de partida de un periplo prolongado y complejo que impulsa su desarrollo profesional (Serrano y Pontes, 2017). Se tiene consciencia, en este sentido, de la importancia que adquiere la capacitación teórico-práctica para el desempeño de una enseñanza comprometida, sensible y capaz de responder a los retos socio-culturales del momento. Una enseñanza que se proyecte sobre el aprendizaje contextualizado, experiencial y personal de los estudiantes y, en definitiva, que garantice su derecho a recibir una educación equitativa y de calidad.

En el marco de la educación inclusiva, las conclusiones son coincidentes. Numerosos estudios indican que uno de los factores determinantes de su materialización es la formación que recibe el profesorado al respecto (Boer, Pijl y Minnaert, 2011; Main, Chambers y Sarah, 2016; Varcoe y Boyle, 2014; Vélez-Calvo, Tárraga-Mínguez, Fernández-Andrés y Sanz-Cervera, 2016). Esto se debe a que, a través de ella, conocen las bases teóricas y los procedimientos para atender a la diversidad de forma adecuada pero, principalmente, porque potencia el desarrollo de actitudes positivas que se derivan, entre otros aspectos, de sentimientos de capacitación para atenderla (Beacham y Rouse, 2012; Conklin, 2012; Hettiarachchi y Das, 2014). Se piensa, asimismo, que tales actitudes podrían impactar posteriormente en el comportamiento, favoreciendo el diseño y el desarrollo de prácticas inclusivas (Azjen, 2014). 
La investigación de Tárraga, Grau y Peirats (2013) pone de manifiesto que el carácter de las actitudes del profesorado hacia la diversidad varía en función de ciertas variables como el sexo, la edad 0 el tipo de necesidad que presente el alumnado. Lo cierto es que existe un conjunto amplio de investigaciones que han centrado su atención en la valoración de las mismas, siendo las posturas identificadas dicotómicas. Mientas algunos estudios son bastante halagüeños (Álvarez y Buenestado, 2015; Colmenero, Pantoja y Pegalajar, 2015), otros subrayan la existencia de actitudes negativas o neutras, así como las inseguridades y la falta de percepción de competencia docente para atender a la diversidad (Boer, Pijl y Minnaert, 2011). También se hallan evidencias que constatan que el alumnado muestra actitudes positivas hacia la diversidad y la inclusión pero afirma no sentirse capacitado para atenderla y hacerla efectiva, lo que redirige las miradas nuevamente hacia los procesos formativos (Hernández, Carrasco, Chiaramello y López, 2012; Tárraga et al., 2013).

En España, las titulaciones requeridas para el ejercicio docente varían en función de las etapas en las que desempeña su labor. En el caso de los docentes de Ed. Infantil y Primaria, los planes de formación - que tienen una duración de cuatro años (Grado) - integran conocimiento científico, didáctico y profesional. De forma distinta, el profesorado de Educación Secundaria recibe primero - en sus estudios de Grado - formación específica de una disciplina determinada y, posteriormente, se forma a nivel didáctico-pedagógico por medio de un postgrado. Esta diferenciación nos lleva a distinguir entre los modelos de formación concurrentes y consecutivos, respectivamente. En este sentido, si bien es cierto que no se ha corroborado que la propuesta concurrente sea más efectiva que la consecutiva, Rebolledo (2015) o Serrano y Pontes (2016) resaltan que esta última puede llegar a crear una falsa identidad, dado que la formación psicopedagógica es posterior y diacrónica respecto a la formación inicial en contenidos disciplinares.

Aunque es cierto que la sustitución, hace casi una década, del Curso de Aptitud Pedagógica por el Máster de Profesorado de Educación Secundaria ha planteado mejoras relevantes, algunos estudios señalan diferencias entre las expectativas y los logros del alumnado en su formación inicial (Serrano y Pontes, 2017), lo que podría hacerse extensible al ámbito de la atención a la diversidad. De hecho, son diversas las investigaciones que plantean que la formación específica sobre este tema es insuficiente (Manso y Martín, 2013; Pegalajar y Colmenero, 2017). Tales afirmaciones podrían justificarse, entre otras razones, por la conclusión a la que llegan en su estudio Tournaki y Ellery (2016), quienes destacan el escaso impacto a largo plazo que tienen los programas breves y puntuales sobre las actitudes. Quizás sea este, además, uno de los factores que podría explicar la afirmación de Tárraga et al. (2013), quienes destacan que, cuanto menor es el nivel educativo en el que trabajan los docentes, más favorable es su actitud hacia la diversidad. Es por ello que este estudio se plantea para dar alcance a los siguientes objetivos:

Conocer las actitudes que posee el profesorado en formación sobre la atención a la diversidad e inclusión educativa.

Valorar sus opiniones sobre el impacto de la formación recibida en su actitud.

Identificar la existencia de diferencias estadísticamente significativas entre los docentes en formación de las etapas de Ed. Infantil/Ed. Primaria y Educación Secundaria, en relación a la variables consideradas.

\section{MÉTODO}

Para dar alcance a los objetivos planteados se optó por desarrollar este estudio desde el enfoque cuantitativo de investigación.

\section{PARTICIPANTES}

Se contó con la participación de 46 estudiantes, matriculados en el último curso de los Grados 


\section{ATENCIÓN A LA DIVERSIDAD Y ESCUELA INCLUSIVA: LAS ACTITUDES DEL FUTURO PROFESORADO}

de Maestro en Educación Infantil y Maestro en Educación Primaria, de la Facultad de Educación, de la Universidad de Alicante (UA). Asimismo, participó un total de 50 alumnos/as del Máster de Profesorado de Educación Secundaria de la citada facultad. En ambos colectivos, la mayoría de los participantes contaba - en el momento del estudio - con una edad comprendida entre los 22 y los 26 años, siendo mayor el porcentaje de alumnas (78\%) entre los estudiantes del primer grupo y estando los porcentajes equiparados en el caso del segundo, en relación a esta variable. Resulta de interés señalar, a su vez, que ninguno de los encuestados de Grado estaba cursando el itinerario de especialización en Pedagogía Terapéutica.

\section{INSTRUMENTO Y PROCEDIMIENTO DE RECOGIDA DE DATOS}

Para la recogida de datos, se recurrió al cuestionario de Colmenero y Pegalajar (2015), diseñado y validado para analizar las actitudes y percepciones sobre atención a la diversidad del alumnado del Máster Universitario en Profesorado de Educación Secundaria. Se seleccionaron siete ítems para los que se propuso una escala de valoración tipo Likert, donde el 1 correspondía a "nada de acuerdo" y el 5 a "totalmente de acuerdo". Cinco de estos ítems se centraban en el reconocimiento de las actitudes hacia la diversidad e inclusión. El resto, por su parte, se enfocaba hacia la valoración del impacto de los planes de estudios en la sensibilización por el tema e interés por la formación al respecto.

El cuestionario se administró vía on-line, a través de un anuncio publicado para las titulaciones citadas en el campus virtual de la UA. En él se informaba a los estudiantes del propósito de la investigación, del anonimato y confidencialidad de la información aportada, así como del carácter voluntario de su participación.

\section{ANÁLISIS ESTADÍSTICOS}

El análisis de los datos se realizó con el soporte del programa SPSS .21. En un primer momento se llevó a cabo un análisis descriptivo, aplicándose con posterioridad la prueba de contraste no paramétrico U de Mann-Whitney. Esta última se llevó a cabo para comprobar la existencia de diferencias estadísticamente significativas en las actitudes del profesorado en formación, en función de su titulación (Grado y Máster), así como para conocer sus opiniones sobre el impacto de la formación en la sensibilización e interés por la formación en este ámbito.

\section{RESULTADOS}

Como puede apreciarse, la Tabla 1 muestra los porcentajes de respuesta de los cinco ítems planteados para conocer las actitudes de los futuros docentes de las etapas de Ed. Infantil y Ed. Primaria. Sus posicionamientos son claros al respecto de los ítems 1,2 y 5 , de lo que se deduce que la totalidad del alumnado piensa que la atención a la diversidad es fuente de enriquecimiento, así como función que asumirá en su futuro desempeño para conseguir hacer efectivo el principio de inclusión. Sus respuestas no están tan polarizadas, sin embargo, en los ítems 3 y 4, en los que opinan sobre si el alumnado con necesidades especiales está mejor atendido en los centros específicos y valoran si su atención en los centros ordinarios supone un trabajo añadido para los docentes. En ambos casos, los mayores porcentajes se localizan en torno al valor 3 de la escala, lo que pone de manifiesto que no tienen una postura claramente definida al respecto. Se comprueba, además, que son los ítems para los que la dispersión entre sus puntuaciones es mayor. 
Tabla 1

Actitudes hacia la diversidad e inclusión de los estudiantes de Grado

\begin{tabular}{|c|c|c|c|c|c|c|c|}
\hline Ítems & 1 & 2 & 3 & 4 & 5 & $M$ & $D T$ \\
\hline $\begin{array}{l}\text { 1.La atención a la diversidad debe } \\
\text { ocupar un papel importante en mi } \\
\text { futura práctica docente }\end{array}$ & $0 \%$ & $0 \%$ & $0 \%$ & $17.4 \%$ & $82.6 \%$ & 4.83 & 0.38 \\
\hline $\begin{array}{l}\text { 2.La atención a la diversidad en el } \\
\text { aula enriquece a toda la comunidad } \\
\text { educativa }\end{array}$ & $0 \%$ & $0 \%$ & $0 \%$ & $6.5 \%$ & $93.5 \%$ & 4.93 & 0.25 \\
\hline $\begin{array}{l}\text { 3.El alumnado con NEAE está } \\
\text { mejor atendido en centros de } \\
\text { educación especial }\end{array}$ & $11.1 \%$ & $31.1 \%$ & $40 \%$ & $13.3 \%$ & $4.4 \%$ & 2.69 & 0.99 \\
\hline $\begin{array}{l}\text { 4.La atención al alumnado con } \\
\text { NEAE es un trabajo añadido para el } \\
\text { profesorado }\end{array}$ & $15.2 \%$ & $23.9 \%$ & $28.3 \%$ & $15.2 \%$ & $17.4 \%$ & 2.96 & 1.31 \\
\hline $\begin{array}{l}\text { 5.Como futuro docente, mi } \\
\text { preocupación será que el alumnado } \\
\text { logre su inclusión en el aula }\end{array}$ & $2.2 \%$ & $0 \%$ & $0 \%$ & $17.4 \%$ & $80.4 \%$ & 4.74 & 0.68 \\
\hline
\end{tabular}

En el caso de la Tabla 2, resulta posible observar las actitudes que muestra el alumnado del Máster hacia estas cuestiones. Sus respuestas son bastante similares a las de los estudiantes del Grado para los ítems 1, 2 y 5, aunque los porcentajes y las medias no llegan a ser tan elevados. Aun así, es obvio que poseen disposición para atender a la diversidad en su futuro desempeño profesional al considerarla un elemento de enriquecimiento. En relación a los ítems para los que el alumnado de Grado no manifestaba un posicionamiento claro (ítems 3 y 4), en este caso se aprecia que las posturas son similares pero no idénticas. Para el primero de los ítems a considerar, se observa que la puntuación media indica que los docentes de las primeras etapas están ligeramente menos de acuerdo con el hecho de que el alumnado con necesidades específicas deba ser atendido en los centros especiales. Ocurre lo mismo en el segundo de los casos, para el que se llega a la conclusión de que el futuro profesorado de Ed. Secundaria parece estar ligeramente más de acuerdo con la afirmación de que la atención a las necesidades específicas en el aula ordinaria supone un trabajo añadido para el docente. Tal y como ocurría en la Tabla 1, se observa que ha habido mayor dispersión entre las respuestas de los participantes a los dos ítems destacados en último término. 


\section{ATENCIÓN A LA DIVERSIDAD Y ESCUELA INCLUSIVA: LAS ACTITUDES DEL FUTURO PROFESORADO}

Tabla 2

Actitudes hacia la diversidad e inclusión de los estudiantes de Máster

\begin{tabular}{lccccccc}
\hline Ítems & 1 & 2 & 3 & 4 & 5 & $M$ & $D T$ \\
\hline $\begin{array}{l}\text { 1.La atención a la diversidad debe } \\
\text { ocupar un papel importante en mi }\end{array}$ & $0 \%$ & $0 \%$ & $6 \%$ & $14 \%$ & $80 \%$ & 4.74 & 0.57 \\
futura práctica docente & & & & & & & \\
$\begin{array}{l}\text { 2.La atención a la diversidad en el } \\
\text { aula enriquece a toda la comunidad } \\
\text { educativa }\end{array}$ & $0 \%$ & $2 \%$ & $6 \%$ & $22 \%$ & $70 \%$ & 4.60 & 0.70 \\
$\begin{array}{l}\text { 3.El alumnado con NEAE está } \\
\text { mejor atendido en centros de }\end{array}$ & $4 \%$ & $36 \%$ & $32 \%$ & $12 \%$ & $16 \%$ & 3.00 & 1.14 \\
educación especial & & & & & & \\
$\begin{array}{l}\text { 4.La atención al alumnado con } \\
\text { NEAE es un trabajo añadido para el }\end{array}$ & $10 \%$ & $22 \%$ & $22 \%$ & $30 \%$ & $16 \%$ & 3.20 & 1.25 \\
profesorado \\
$\begin{array}{l}\text { 5.Como futuro docente, mi } \\
\text { preocupación será que el alumnado } \\
\text { logre su inclusión en el aula }\end{array}$
\end{tabular}

En relación a la opinión que tienen los encuestados sobre el impacto de la formación recibida, la Tabla 3 indica que la mayoría opina que sus estudios de Grado contribuyen al incremento de su sensibilidad por la diversidad. Asimismo, afirman que consigue despertar un interés por recibir mayor formación al respecto.

Tabla 3

Opiniones del alumnado de los Grados sobre el impacto de la formación recibida

\begin{tabular}{|c|c|c|c|c|c|c|c|}
\hline Ítems & 1 & 2 & 3 & 4 & 5 & $M$ & $D T$ \\
\hline $\begin{array}{l}\text { 1.Mis estudios de Grado me están } \\
\text { sirviendo para tener una mayor } \\
\text { sensibilización hacia la atención a la } \\
\text { diversidad }\end{array}$ & $0 \%$ & $4.3 \%$ & $13 \%$ & $37 \%$ & $45.7 \%$ & 4.24 & 0.85 \\
\hline $\begin{array}{l}\text { 2.Mis estudios de Grado me están } \\
\text { sirviendo para aumentar mi interés } \\
\text { hacia una mayor formación en el } \\
\text { ámbito de la atención a la diversidad }\end{array}$ & $2.2 \%$ & $2.2 \%$ & $13 \%$ & $43.5 \%$ & $39.1 \%$ & 4.15 & 0.89 \\
\hline
\end{tabular}

Si tratamos de comparar estos resultados con los del futuro profesorado de Ed. Secundaria (Tabla 4), se concluye que las puntuaciones en ambos casos son ligeramente inferiores. Sin embargo, los porcentajes más altos continúan localizándose en los valores 4 y 5 de la escala, lo que indica que - aunque en menor medida que los estudiantes del Grado - valoran su formación por cuanto favorece su sensibilización con la temática, motivándoles a continuar formándose en la materia. 
Tabla 4

Opiniones del alumnado del Máster sobre el impacto de la formación recibida

\begin{tabular}{|c|c|c|c|c|c|c|c|}
\hline Ítems & 1 & 2 & 3 & 4 & 5 & $M$ & $D T$ \\
\hline $\begin{array}{l}\text { 1.Mis estudios de Grado me están } \\
\text { sirviendo para tener una mayor } \\
\text { sensibilización hacia la atención a la } \\
\text { diversidad }\end{array}$ & $6 \%$ & $0 \%$ & $22 \%$ & $34 \%$ & $38 \%$ & 3.98 & 1.08 \\
\hline $\begin{array}{l}\text { 2.Mis estudios de Grado me están } \\
\text { sirviendo para aumentar mi interés } \\
\text { hacia una mayor formación en el } \\
\text { ámbito de la atención a la diversidad }\end{array}$ & $8 \%$ & $10 \%$ & $24 \%$ & $30 \%$ & $28 \%$ & 3.60 & 1.22 \\
\hline
\end{tabular}

En cuanto al resultado de la aplicación de la prueba no paramétrica U de Mann-Whitney, se comprueba la existencia de diferencias estadísticamente significativas para el ítem 2 del apartado de actitudes (La atención a la diversidad en el aula enriquece a toda la comunidad educativa) y para el ítem 2 del apartado de impacto de la formación recibida (utilidad de los estudios para aumentar el interés hacia una mayor formación en el ámbito de la atención a la diversidad). En el primero de los casos, la U tiene un valor de 874, al que le corresponde una significación de 0.00 . Por su parte, en el segundo de los casos, la U posee un valor de 857, al que le corresponde una significación de 0.02 . La existencia de estas diferencias nos lleva a considerar los rangos promedio para ambos ítems y se comprueba que son mayores - en ambos casos - para el alumnado de los Grados, lo que indica que este colectivo reconoce más favorablemente en la diversidad un elemento de enriquecimiento de la comunidad. Asimismo, se deduce que el futuro profesorado de Ed. Infantil y Ed. Primaria considera que su formación le anima a incrementar, en mayor medida, el interés por su capacitación en este ámbito.

\section{DISCUSIÓN Y CONCLUSIONES}

A la luz de los resultados obtenidos, se puede llegar a afirmar que el profesorado en formación, de una etapa y otra, posee actitudes positivas hacia la atención a la diversidad y la inclusión. Ciertamente, la literatura científica, ha evidenciado la existencia de actitudes positivas y negativas entre los participantes a este respecto (Álvarez y Buenestado, 2015; Boer, Pijl y Minnaert, 2011; Tárraga et al., 2013). En este caso, sin embargo, las conclusiones son concomitantes con aquellos hallazgos que destacan una predisposición y actitud favorable hacia la diversidad y la escuela para todos/as (Álvarez y Buenestado, 2015; Colmenero et al., 2015; Pegalajar y Colmenero, 2017). Aun así es interesante destacar que, si bien ambos colectivos poseen actitudes favorables en las cuestiones más generales, cuando tienen que opinar sobre aspectos que indirectamente cuestionan su posicionamiento, la neutralidad llega a ser la tónica. Estas opiniones no son congruentes con las tesis de algunos estudios que ponen de manifiesto que: (1) el alumnado con necesidades específicas obtiene mejores resultados cuando participa en el aula ordinaria (Ruijs, Can der Veen and Peetsma, 2010); y, (2) que enseñar a niños/as con discapacidad no supone necesariamente desarrollar una práctica distinta pero sí efectiva (Foreman, 2011; Killoran et al., 2014).

Por otra parte, si bien es cierto que los estudiantes de Grado y Máster encuestados muestran, a nivel general, actitudes positivas hacia este tópico, no lo es menos que los primeros obtienen puntuaciones reveladoras de una actitud ligeramente más favorable. Incluso las diferencias entre ambos colectivos llegan a ser significativas a la hora de valorar la atención a la diversidad como elemento enriquecedor del conjunto de la comunidad. Esto podría guardar relación con las conclusiones a las que llegan Tárraga et al. (2013), en las que destacan que cuanto menor es el nivel educativo en el 


\section{ATENCIÓN A LA DIVERSIDAD Y ESCUELA INCLUSIVA: LAS ACTITUDES DEL FUTURO PROFESORADO}

que trabaja el profesorado, más favorable es su actitud hacia la diversidad, lo que podría relacionarse también con las aportaciones de Tournaki y Ellery (2016), toda vez que parece que el Máster, por su brevedad, trabajaría de forma más puntual y rápida esta temática, lo que podría producir un impacto a corto plazo en el cambio de actitudes y un incremento en la sensación de los estudiantes de falta de capacitación para hacer efectiva la inclusión (Hernández-Amorós y Urrea-Solano, 2016).

En última instancia, se aprecia que los encuestados subrayan la utilidad de sus estudios como vía de sensibilización con el tema e impulso de su interés para continuar formándose al respecto (Colmenero et al., 2015), lo que refuerza el planteamiento de que la formación incide sobre las actitudes del profesorado (Beacham y Rouse, 2012; Conklin, 2012; Hettiarachchi y Das, 2014). A este respecto, curiosamente, se aprecia que una vez más los futuros docentes de las primeras etapas están más convencidos del potencial de la formación en este sentido, quizás por el hecho de que aunque el Máster ha ampliado los conocimientos psicopedagógicos y didácticos, así como la duración de las prácticas, no se considera suficiente para forjar una identidad docente ajustada a las nuevas demandas educativas (Serrano y Pontes, 2016).

En conclusión, aunque las actitudes de los estudiantes de los Grados y el Máster son positivas, es necesario continuar trabajando sobre ellas - especialmente en el Máster - para favorecer un desempeño profesional, totalmente comprometido con la inclusión en los escenarios prácticos. De igual modo, convendría seguir investigando sobre la utilidad de los planes de formación, no únicamente para aumentar la sensibilización sobre el tema - lo que corrobora que la formación sí incide sobre el cambio actitudinal - sino para valorar la percepción de competencia que tiene el profesorado al respecto. Se ha comprobado, en otros estudios, que a pesar de que las actitudes suelen ser positivas, los futuros docentes de unas etapas y otras consideran que su formación para la educación inclusiva es claramente insuficiente (Pegalajar y Colmenero, 2017; Vélez-Calvo et al., 2016). Futuros estudios podrían centrarse en este aspecto, así como en hallar posibles diferencias en las actitudes del alumnado de los propios Grados (Infantil y Primaria), analizándolas tanto desde un prisma cuantitativo como cualitativo de investigación.

\section{REFERENCIAS}

Álvarez, J.L. y Buenestado, M. (2015). Predictores de las actitudes hacia la inclusión de alumnado con necesidades educativas especiales en futuros profesionales de la educación. Revista Complutense de Educación, 26(3), 627-645. Doi: 10.5209/rev_RCED.2015.v26.n3.44551

Azjen, I. (2015). The theory of planned behaviour is alive and well, and not ready to retire: a commentary on sniehotta, Presseau, and Araújo-Soares. Health Psychology Review, 9(2), 131-137. Doi: 10.1080/17437199.2014.883474

Beacham, N. y Rouse, M. (2012). Student teachers' attitudes and beliefs about inclusion and inclusive practice. Journal of Research in Special Educational Needs, 12(1), 3-11. Doi: 10.1111/j.1471-3802.2010.01194.x

Boer, A.A., Pijl, S.J. y Minnaert, A.E. (2011). Regular primary school teachers' attitudes towards inclusive education: A review of the literature. International Journal of Inclusive Education, 15(3), 331-353. Doi: 10.1080/13603110903030089

Colmenero, M.J. y Pegalajar, M.C. (2015). Cuestionario para futuros docentes de Educación Secundaria acerca de las percepciones sobre atención a la diversidad: Construcción y validación del instrumento. Estudios sobre Educación, 29(1), 165-189. Doi: 10.15581/004.29.165-189

Colmenero, M.J., Pantoja, A. y Pegalajar, M.C. (2015). Percepciones del alumnado sobre atención a la diversidad en la formación inicial del profesorado de Educación Secundaria. Revista Complutense de Educación, 26(1), 101-120. Doi: 10.5209/rev_RCED.2015.v26.n1.42616 
Conklin, H. (2012). Tracing learning from divergent teacher education pathways into practice in middle grades classrooms. Journal of Teacher Education, 63(3), 171-184. Doi: $10.1177 / 0022487111426294$

Foreman, P. (2011). Inclusion in action. Melbourne: Cengage.

Hernández, M.J., Carrasco, V., Chiaramello, G. y López, C. (2012). Diversidad e inclusión: percepciones de los estudiantes del Grado de Maestro en Educación Primaria. Educación y Diversidad, 6(2), 69-84.

Hernández-Amorós, M.J. y Urrea-Solano, M. (2016). Pre-service secondary teachers' attitudes and training towards diversity. En L. Gómez, A. López e I. Candel (Eds.), EDULEARN16. 8th International conference on education and new learning technologies. Barcelona: IATED.

Hettiarachchi, S. y Das, A. (2014). Perceptions of inclusion and perceived preparedness among school teachers in Sri Lanka. Teaching and Teacher Education, 43, 143-153. Doi: 10.1016/j.tate.2014.07.003

Killoran, I., Woronko, D. y Zaretsky, H. (2014). Exploring preservice teachers' attitudes towards inclusion. International Journal of Inclusive Education, 18(4), 427-442. Doi: 10.1080/13603116.2013.784367

Main, S., Chambers, D.J. y Sarah, P. (2016). Supporting the transition to inclusive education: teachers' attitudes to inclusion in the Seychelles. International Journal of Inclusive Education, 20(12). Doi: 10.1080/13603116.2016.1168873

Manso, J. y Martín, E. (2014). Valoración del Máster de Formación de Profesorado de Educación Secundaria: estudio de casos en dos universidades. Revista de Educación, 364, 145-169. Doi: 10.4438/1988-592X-RE-2014-364-258

Pegalajar, M.C., y Colmenero, M.J. (2017). Actitudes y formación docente hacia la inclusión en Educación Secundaria Obligatoria. REDIE, Revista Electrónica de Investigación Educativa, 19(1), 84-97.

Rebolledo, T. (2015). La formación inicial del profesorado de Educación Primaria y Secundaria en Alemania, España, Finlandia, Francia y Reino Unido. Estudio comparado. Revista Española de Educación Comparada, 25, 129-148. Doi: 10.5944/reec.25.2015.14787

Ruijs, N. M., Van der Veen, I. y Peetsma, T. T. D. (2010). Inclusive education and students without special educational needs. Educational Research, 52(4), 351-390. Doi:10.1080/00131881.2010.524749

Serrano, R. y Pontes, A. (2016). El desarrollo de la identidad profesional docente en la formación inicial del profesorado de Secundaria. Enseñanza \& Teaching, 34(1), 35-55. Doi: 10.14201/et20163413555

Serrano, R. y Pontes, A. (2017). Diferencias entre expectativas y logros en la formación inicial del profesorado de secundaria. Revista Complutense de Educación, 28(3), 791-807. Doi: 10.5209/rev_RCED.2017.v28.n3.49856

Tárraga, R., Grau, C. y Peirats, J. (2013). Actitudes de los estudiantes del Grado de Magisterio y del Máster de Educación Especial hacia la inclusión educativa. Revista Electrónica Interuniversitaria de Formación del Profesorado, 16(1), 55-72. Doi: 10.6018/reifop.16.1.179441

Tournaki, N. y Ellery, W. (2016). Do graduate teacher education programs change teachers' attitudes toward inclusion and efficacy beliefs? Action in Teacher Education, 38(4), 384-398. Doi: $10.1080 / 01626620.2016 .1226200$

Valle, J. y Manso, J. (2013). Competencias clave como tendencia de la política educativa supranacional de la Unión Europea. Revista de Educación, (extraordinario), 12-33. Doi: 10.4438/1988592X-RE-2013-EXT-255 
Varcoe, L. y Boyle, C. (2014). Pre-service primary teachers' attitudes towards inclusive education. Educational Psychology, 34(3), 323-337. Doi: 10.1080/01443410.2013.785061

Vélez-Calvo, X., Tárraga-Mínguez, R., Fernández-Andrés, M.I. y Sanz-Cervera, P. (2016). Formación inicial de maestros en Educación Inclusiva: una comparación entre Ecuador y España. Revista Nacional e Internacional de Educación Inclusiva, 9(3), 75-94. 